\title{
Outcomes and prognostic stratification of patients with recurrent glioblastoma treated with salvage stereotactic radiosurgery
}

\author{
Mayur Sharma, MD, ${ }^{1}$ Jason L. Schroeder, MD, ${ }^{2}$ Paul Elson, ScD, ${ }^{3}$ Antonio Meola, MD, ${ }^{1}$ \\ Gene H. Barnett, MD, ${ }^{1}$ Michael A. Vogelbaum, MD, PhD, John H. Suh, MD, ${ }^{4}$ Samuel T. Chao, MD, ${ }^{4}$ \\ Alireza M. Mohammadi, MD, ${ }^{1}$ Glen H. J. Stevens, DO, PhD, ${ }^{1}$ Erin S. Murphy, MD, ${ }^{4}$ and \\ Lilyana Angelov, MD'
}

\begin{abstract}
${ }^{1}$ The Rose Ella Burkhardt Brain Tumor and Neuro-Oncology Center, Department of Neurosurgery, Neurological Institute, Cleveland Clinic, Cleveland; '2Department of Surgery, Division of Neurosurgery, University of Toledo Medical Center, Toledo; ${ }^{3}$ Department of Quantitative Health Sciences, Cleveland Clinic; and ${ }^{4}$ The Rose Ella Burkhardt Brain Tumor and Neuro-Oncology Center, Department of Radiation Oncology, Cleveland Clinic, Cleveland, Ohio
\end{abstract}

OBJECTIVE Glioblastoma (GBM) is the most malignant form of astrocytoma. The average survival is $6-10$ months in patients with recurrent GBM (rGBM). In this study, the authors evaluated the role of stereotactic radiosurgery (SRS) in patients with rGBMs.

METHODS The authors performed a retrospective review of their brain tumor database (1997-2016). Overall survival (OS) and progression-free survival (PFS) after salvage SRS were the primary endpoints evaluated. Response to SRS was assessed using volumetric MR images.

RESULTS Fifty-three patients with rGBM underwent salvage SRS targeting 75 lesions. The median tumor diameter and volume were $2.55 \mathrm{~cm}$ and $3.80 \mathrm{~cm}^{3}$, respectively. The median prescription dose was $18 \mathrm{~Gy}$ (range 12-24 Gy) and the homogeneity index was 1.90 (range 1.11-2.02). The median OS after salvage SRS was estimated to be 11.0 months (95\% $\mathrm{CI} 7.1-12.2)$ and the median PFS after salvage SRS was 4.4 months (95\% CI 3.7-5.0). A Karnofsky Performance Scale score $\geq 80$ was independently associated with longer OS, while small tumor volume $\left(<15 \mathrm{~cm}^{3}\right)$ and less homogeneous treatment plans (homogeneity index $>1.75$ ) were both independently associated with longer OS ( $p=0.007$ and 0.03 ) and PFS ( $p=0.01$ and 0.002 , respectively). Based on these factors, 2 prognostic groups were identified for PFS (5.4 vs 3.2 months), while 3 were identified for OS (median OS of 15.2 vs 10.5 vs 5.2 months).

CONCLUSIONS SRS is associated with longer OS and/or PFS in patients with good performance status, small-volume tumor recurrences, and heterogeneous treatment plans. The authors propose a prognostic model to identify a cohort of rGBM patients who may benefit from SRS.

https://thejns.org/doi/abs/10.3171/2018.4.JNS172909

KEYWORDS recurrent GBM; stereotactic radiosurgery; brain tumor; glioblastoma; Gamma Knife; salvage treatment; oncology

$\mathrm{G}$ LIOBLASTOMA (GBM) is the most malignant subtype constituting approximately $55 \%$ of all glial brain tumors. ${ }^{10,31}$ Despite aggressive management, these tumors tend to recur within 6 months of treatment initiation, and the prognosis remains dismal. ${ }^{52,54}$

Patients with recurrent GBM (rGBM) pose significant clinical management challenges, as no standard salvage treatment is currently available for these patients. ${ }^{42} \mathrm{Op}-$ tions such as repeat resection, ${ }^{57,58}$ laser interstitial thermal therapy (LITT), ${ }^{46,47,60}$ repeat external-beam radiotherapy (EBRT) alone ${ }^{6,40}$ or in combination with other agents, ${ }^{1,28}$ salvage stereotactic radiosurgery (SRS), ${ }^{3,8,32,33,61}$ various

ABBREVIATIONS EBRT = external-beam radiotherapy; GBM = glioblastoma; GKRS = Gamma Knife radiosurgery; KPS = Karnofsky Performance Scale; LITT = laser interstitial thermal therapy; MGMT = $0^{6}$-methylguanine-DNA methyltransferase; $\mathrm{OS}=$ overall survival; $\mathrm{PFS}=$ progression-free survival; $\mathrm{rGBM}=$ recurrent $\mathrm{GBM}$; $\mathrm{RPA}=$ recursive partitioning analysis; SRS = stereotactic radiosurgery; WBRT = whole-brain radiation therapy.

SUBMITTED November 17, 2017. ACCEPTED April 23, 2018.

INCLUDE WHEN CITING Published online October 19, 2018; DOI: 10.3171/2018.4.JNS172909. 
chemotherapeutic agents, ,,13,35,59,65-67 targeted therapies, $, 2,7$, 13,23,38 immunotherapeutic options, ${ }^{15,37,41}$ and tumor treating fields ${ }^{53,55,56}$ have all been utilized with varied success. Salvage radiation with SRS is a well-established treatment modality for a variety of benign ${ }^{17,19,24,64}$ and malignant brain tumors. ${ }^{11,16,21,34,39,50,62,68}$ There is, however, insufficient evidence to either support or refute the use of radiosurgery in patients with recurrent or progressive malignant glioma; ${ }^{63}$ various salvage SRS series have reported a median overall survival (OS) ranging between 5.3 and 24 months in patients with rGBM. ${ }^{3,30,32,33,49}$ The majority of these series are small retrospective studies with limited and heterogeneous patient populations.

In this study, we present our single-center 20-year experience with salvage SRS in managing 53 patients (75 lesions) with rGBM. We also propose a prognostic model to identify a cohort of patients with favorable factors who may benefit from SRS.

\section{Methods}

A retrospective review of the IRB-approved brain tumor database (1997-2016) was performed following IRB approval. Patients with no clinical follow-up or those who were enrolled in another clinical trial at our center investigating dose escalation of SRS in the setting of bevacizumab therapy for $\mathrm{rGBM}(\mathrm{n}=9)$ were excluded from this study.

\section{Variables, Participants, and Data Source}

Patients' electronic medical records were retrospectively reviewed to obtain demographic, imaging, and clinical data. Tumor-related variables (maximum tumor dimension, tumor volume, tumor location) and SRS data (prescription dose, maximum dose, conformity index [prescription isodose volume/tumor volume], homogeneity index [maximum dose/prescription tumor dose], gradient index) were also collected. Data from 53 patients (75 lesions) who underwent SRS for rGBM as a salvage therapy were included.

Molecular evaluation from the initial surgical specimens was performed with immunohistochemical staining with antibodies to IDH1 (R132H), Tp53, and Ki-67 to identify the IDH1 mutation and percentage of tumor cells staining positive for $\mathrm{Tp} 53$ and $\mathrm{Ki}-67$. The percentages of tumor cells staining positive to Tp53 antibodies were segregated as $<10 \%, 10 \%-32 \%, 33 \%-66 \%$, and $>66 \%$, as previously described. ${ }^{29}$ Similarly, tumor cells staining positive to $\mathrm{Ki}-67$ antibodies were classified as $<10 \%$, $10 \%-30 \%$, and $>30 \% .{ }^{69} \mathrm{O}^{6}$-methylguanine-DNA methyltransferase (MGMT) methylation status was determined using standard polymerase chain reaction (PCR) amplification of a targeted region of exon 1, and methylation of CpG sites was analyzed by pyrosequencing of the amplicons using the PyroMark Q96 ID instrument (Qiagen). The mean percentage of methylation was calculated and used to determine methylation status. Amplification of the EGFR gene was evaluated using interphase fluorescence in situ hybridization (FISH) on formalin-fixed, paraffinembedded tissue sections using epidermal growth factor receptor (EGFR; 7p12) and chromosome 7 pericentro- meric (7p11.1-q11.1) locus-specific directly labeled probes (Abbott Molecular/Vysis).

\section{Gamma Knife Radiosurgery}

SRS was delivered using Gamma Knife radiosurgery (GKRS; Elekta AB) at our center, as described in our previous studies. ${ }^{43,44}$ As the present series spans 20 years, GKRS was performed using different models-B, C, 4C, and Perfexion-during the study period. Since 2007, we have been using the Perfexion model for GKRS. GKRS is performed as an outpatient procedure following acquisition of informed consent. Briefly, on the day of the procedure, a stereotactic contrast-enhanced T1-weighted magnetization-prepared rapid acquisition gradient echo (MPRAGE) MRI study (multiplanar reconstruction sequence with 2- to 3-mm-thick slices, no interslice gaps, and isotropic $1.0-\mathrm{mm}$ voxels) was obtained either before or after Leksell frame (Elekta AB) placement. A postframe, stereotactic CT scan was then obtained and coregistered to the volumetric MR image at the planning station.

\section{Target Definition and Clinical Follow-Up}

Following satisfactory image coregistration, target tumor volume was defined as the contrast-enhanced tumor margin on the volumetric contrast-enhanced T1-weighted MRI without including the T2-weighted/FLAIR leading edge. A conformal plan was created using a combination of shots. The prescription dose was determined based on the maximum dimension of the tumor, according to the Radiation Therapy Oncology Group (protocol 90-05) guidelines. ${ }^{45}$ Following GKRS, 4-6 weeks after the procedure, patients underwent clinical examination and MRI and then every 3 months thereafter. The median followup duration in our study was 10.1 months (range $0.8-51.4$ months).

\section{Outcomes and Endpoints}

Overall survival (OS) and progression-free survival (PFS) from the date of salvage SRS were the primary and secondary endpoints, respectively. OS and PFS were measured from the time of salvage SRS to death and the first instance of tumor progression/recurrence or death, respectively.

Radiographic response to SRS was assessed using RANO criteria on contrast-enhanced T1-weighted and T2-weighted FLAIR MR images. ${ }^{27}$ Also, the response to therapy was evaluated using volumetric analysis, after importing the post-SRS MR images into iPlan software (Brainlab AG), and findings were compared to those demonstrated on the pre-SRS MR images. Radiation necrosis was defined based on radiographic findings and our multidisciplinary brain tumor board consensus input, as previously described..$^{5,43,44}$ Additionally, MR perfusion images or short-term follow-up MR images (4-6 weeks) were used to differentiate between radiation necrosis and local tumor progression in certain cases.

\section{Statistical Analysis}

The log-rank test (univariable) and proportional hazards model (univariable and multivariable) were used to 
analyze the data. Stepwise variable selection, with $p=0.10$ and 0.05 as the criteria for entry and retention in the model, was used to determine which, if any, provided independent prognostic information. Patients treated in 2005 and earlier $(n=23$ [43\%]) (i.e., prior to the introduction of Stupp's protocol featuring the survival benefit of first-line temozolomide in patients with $\mathrm{GBM}^{54}$ ) had a worse outcome than patients treated after 2005 (median OS 10.0 vs 11.8 months, $\mathrm{p}=0.05$; median PFS 4.1 vs 4.5 months, $\mathrm{p}=0.05$ ). Therefore, to account for inherent differences related to when the patient was treated, the multivariable models were stratified by the year SRS was performed (2005 and earlier or after 2005). For convenience, recursive partitioning analysis (RPA) was used to find cut-points for continuous variables. Some patients had more than one lesion treated in a single session, and since variables such as maximum dimension, volume, progression, and SRS parameters are specific to the lesion, two analyses were performed. A lesion-specific analysis was performed in which all lesions were considered as separate "cases" and the marginal Cox model approach was used to account for the correlation that might exist between lesions within the same patient. ${ }^{25}$ However, since most patients had a single lesion, a second, patient-specific analysis was also performed that aggregated lesion and SRS parameters. Statistical analyses were performed using SAS 9.4 (SAS Institute, Inc.).

\section{Results}

\section{Participants and Descriptive Data}

A majority of patients $(n=52,98.1 \%)$ were treated using single-stage SRS and 11 patients $(21 \%)$ had multiple lesions (4 patients [7.5\%] underwent treatment in 2 sessions for different lesions and 1 patient [1.8\%] underwent treatment in 3 stages for a single lesion [12 Gy per fraction]). Most of the patients $(70 \%, \mathrm{n}=34)$ had good performance status (Karnofsky Performance Scale [KPS] score $\geq 80)$. Also, most patients were RPA class $4(47 \%, n=23)$ or classes 5 and $6(43 \%, \mathrm{n}=21)^{26}$ (Table 1). The decrease was $>25 \%$ in 15 of 36 lesions $(42 \%)$ and $>50 \%$ in 8 of $36(22 \%)$.

Since the series spans 2 decades, molecular analysis was only available for patients treated in the later part of the series (since 2009). The majority $(87.5 \%, \mathrm{n}=14 / 16)$ of patients with data had wild-type IDH, $35 \%(n=7 / 20)$ had elevated p53 staining $(\geq 33 \%), 41 \%(\mathrm{n}=13 / 32)$ had elevated Ki-67 staining $(>30 \%)$, and $66.7 \%(n=10 / 15)$ had MGMT-unmethylated GBM.

\section{Lesion Characteristics}

The median maximum tumor dimension and volume were $2.55 \mathrm{~cm}$ (range $0.25-6.90 \mathrm{~cm}$ ) and $3.80 \mathrm{~cm}^{3}$ (range 0.01-29.70 $\mathrm{cm}^{3}$ ), respectively. The median prescription dose was 18 Gy (range 12-24 Gy), and the majority of lesions $(46 \%)$ were treated at a dose of $15 \mathrm{~Gy}$ to the tumor margin. The median conformity index was 1.85 (range 1.35-13.27), and the median homogeneity index was 1.90 (range 1.11-2.02).

Most lesions $(74 \%, 56 / 75)$ were initially treated with either gross-total tumor resection $(57 \%, 43 / 75)$ or subtotal resection $(17 \%, 13 / 75)$ followed by chemoradiotherapy
TABLE 1. Patient, disease, and treatment characteristics, on per-patient basis

\begin{tabular}{|c|c|}
\hline Factor (range) & No. $(\%)$ or Median (range) \\
\hline \multicolumn{2}{|l|}{ Sex } \\
\hline Female & $17(32 \%)$ \\
\hline Male & $36(68 \%)$ \\
\hline Age at SRS, yrs ${ }^{*} \dagger$ & $58(19-82)$ \\
\hline \multicolumn{2}{|l|}{ KPS score*t‡ } \\
\hline $50-60$ & $4(8 \%)$ \\
\hline 70 & $11(22 \%)$ \\
\hline 80 & $19(39 \%)$ \\
\hline $90-100$ & $15(31 \%)$ \\
\hline Interval from diagnosis to SRS, $\operatorname{mos}^{*} \dagger$ & $9.0(0.07-71.5)$ \\
\hline \multicolumn{2}{|l|}{ RPA class ${ }^{*}$} \\
\hline Class 3 & $5(10 \%)$ \\
\hline Class 4 & $23(47 \%)$ \\
\hline Classes 5 \& 6 & $21(43 \%)$ \\
\hline \multicolumn{2}{|l|}{$\%(+) \operatorname{Tp} 53^{*} \S$} \\
\hline$<10 \%$ & $6(30 \%)$ \\
\hline $10-32 \% \S$ & $7(35 \%)$ \\
\hline $33-66 \%$ & $2(10 \%)$ \\
\hline$>66 \%$ & $5(25 \%)$ \\
\hline \multicolumn{2}{|l|}{ IDH mutation* } \\
\hline Negative & $14(87.5 \%)$ \\
\hline Positive & $2(12.5 \%)$ \\
\hline \multicolumn{2}{|l|}{ EGFR amplification* } \\
\hline No & $11(50 \%)$ \\
\hline Yes & $11(50 \%)$ \\
\hline \multicolumn{2}{|l|}{ Percentage of Ki-67-positive-stained cells* } \\
\hline$<10 \%$ & $5(15.6 \%)$ \\
\hline $10-30 \%$ & $14(43.7 \%)$ \\
\hline$>30 \%$ & $13(40.6 \%)$ \\
\hline \multicolumn{2}{|l|}{ MGMT methylation* } \\
\hline No & $10(66.7 \%)$ \\
\hline Yes & $5(33.3 \%)$ \\
\hline
\end{tabular}

* Missing data: age $(n=1)$; KPS score $(n=4) ; \operatorname{RPA}(n=4)$; interval from diagnosis $(n=3) ;$ p53 ( $n=34)$; IDH $(n=37)$; EGFR $(n=31)$; Ki-67 $(n=21)$; and MGMT $(n=38)$

† First SRS if $>1$ session.

$\ddagger$ In the series, 2 patients had a KPS score of 50,2 had a score of 60 , and 2 had a score of 100 .

$\S$ This translates into percentage of tumor cells staining positive for Tp53.

prior to salvage SRS. One patient with one lesion (1.3\%) had LITT prior to SRS. Most lesions were treated with at least two additional treatment modalities following the index procedure prior to salvage SRS $(56 \%, 42 / 75)$. Considering all treatments after the index procedure but prior to salvage SRS, most lesions at some point were treated with EBRT and chemotherapy, with $(8 \%, 6 / 75)$ or without $(56 \%$, 42/75) additional surgery. Seven percent (5/75) of lesions were treated with whole-brain radiation therapy (WBRT) only, and 5\% (4/75) were treated with WBRT and chemo- 
therapy with or without additional surgery (Table 2). Also, 3 lesions developed radiation necrosis $(3 / 75,4 \%)$ following SRS during the follow-up period with no focal neurological deficits that could be attributed to radiation necrosis. None of the patients who received EBRT following SRS developed radiation necrosis during the follow-up period. These patients with radiation necrosis were treated using conservative management.

\section{Outcome Data, Main Results, and Other Analyses Overall Survival}

Overall, 83\% (44/53) of patients died, and the median OS was estimated to be 11.0 months (95\% CI 7.1-12.2). Smaller tumor volume $\left(<15 \mathrm{~cm}^{3}, \mathrm{p}=0.03\right)$, low isodose line $(\leq 60, p=0.03)$, and higher homogeneity index $(>1.75$, $\mathrm{p}=0.09)$ were associated with improved OS in the univariable analysis, as was KPS (score $\geq 80, p=0.0007$ ) and RPA (class 3 vs 4 vs 5 or $6, p=0.07$ ). However, in multivariable analysis, only total tumor volume, KPS score, and homogeneity index (i.e., more heterogeneous plans) were found to be independent predictors of OS ( $p$ $=0.007,0.0004$, and 0.03, respectively) (Tables 3 and 4 and Fig. 1). Among the subset of patients with molecular data, those with $\geq 10 \%$ of cells staining positive for $\mathrm{p} 53$ or $\mathrm{Ki}-67$ had longer OS compared to those with $<10 \%$ (13.0 vs 5.4 months for $\mathrm{p} 53, \mathrm{p}=0.008$; 11.8 vs 5.5 months for $\mathrm{Ki}-67, \mathrm{p}=0.04)$. However, neither factor was identified as an independent prognostic factor for OS ( $p 53: \mathrm{p}=0.57$; Ki-67: $\mathrm{p}=0.95$ ) in the multivariable analysis.

\section{Progression-Free Survival}

Six-month survival and median PFS were estimated to be $28 \% \pm 6 \%$ and 4.4 months $(95 \%$ CI $3.7-5.0)$, respectively. Similar to OS, total tumor volume $\left(<15 \mathrm{~cm}^{3}, \mathrm{p}=\right.$ $0.03)$, low treatment isodose line $(\leq 60, p=0.004)$, and high homogeneity index $(>1.75, \mathrm{p}=0.02)$ were significantly associated with improved PFS in the univariable analysis, as was smaller tumor diameter $(<3 \mathrm{~cm}, \mathrm{p}=0.03)$. In multivariable analysis, only smaller total tumor volume $(p=0.01)$ and high homogeneity index $(p=0.002)$ were found to be independent predictors of PFS. None of the molecular markers were associated with PFS on univariable analysis ( $p>0.05$ ) (Tables 3 and 4 and Fig. 1).

\section{Prognostic Groups for OS and PFS}

For OS, in addition to tumor volume and homogeneity index, KPS score was also seen to be an independent prognostic factor. By simply counting the number of poor features present (total volume $\geq 15 \mathrm{~cm}^{3}$, homogeneity index [minimum if $>1$ lesion treated] $\leq 1.75$, and KPS score $<80$ ), 4 prognostic groups were possible; however, only 1 patient had all 3 poor prognostic features (OS was 5.2 months), and therefore the last 2 groups were combined. The 3 resulting prognostic groups were labeled "favorable," "intermediate," and "unfavorable" and comprised $39 \%$ (17/44), $50 \%$ (22/44), and $11 \%$ (5/44) of patients, respectively. The estimated median OS durations for the 3 groups were 15.2 months (95\% CI 7.4-29.0 months), 10.5 months (95\% CI 5.2-12.2 months), and 5.2 months (95\% CI 0.8-6.3 months), respectively (Table 5 and Fig. 2).
TABLE 2. Patient, disease, and treatment characteristics based on per-lesion analysis

\begin{tabular}{|c|c|}
\hline Factor (range) & No. (\%) or Median (range) \\
\hline Prescription dose, $\mathrm{Gy}^{*} \dagger$ & $18(12-24)$ \\
\hline 15 & $34(46 \%)$ \\
\hline 18 & $16(22 \%)$ \\
\hline 24 & $24(32 \%)$ \\
\hline Isodose line & $52(50-90)$ \\
\hline Conformity* & $1.85(1.00-13.27)$ \\
\hline Homogeneity* & $1.90(1.11-2.02)$ \\
\hline Longest diameter, $\mathrm{cm}$ & $2.55(0.25-6.90)$ \\
\hline Volume, $\mathrm{cm}^{3 *}$ & $3.80(0.01-29.70)$ \\
\hline \multicolumn{2}{|l|}{ Initial treatment $\ddagger$} \\
\hline Biopsy & $5(7 \%)$ \\
\hline GTR & $43(57 \%)$ \\
\hline STR & $13(17 \%)$ \\
\hline NeuroBlate & $1(1.3 \%)$ \\
\hline SRS & $2(3 \%)$ \\
\hline \multicolumn{2}{|c|}{$\begin{array}{l}\text { No. of subsequent treatments prior to } \\
\text { salvage SRS } \S\end{array}$} \\
\hline 1 & $23(31 \%)$ \\
\hline 2 & $21(28 \%)$ \\
\hline 3 & $10(13 \%)$ \\
\hline$>3 \pi$ & $11(15 \%)$ \\
\hline \multicolumn{2}{|c|}{ Modalities of subsequent procedures } \\
\hline Any surgery§ & $16(21 \%)$ \\
\hline Any EBRT & $55(73 \%)$ \\
\hline Any WBRT & $10(13 \%)$ \\
\hline Any systemic treatment & $58(77 \%)$ \\
\hline \multicolumn{2}{|l|}{ Overall procedures ${ }^{* *}$} \\
\hline EBRT only & $4(5 \%)$ \\
\hline WBRT only & $5(7 \%)$ \\
\hline Systemic only & $1(1 \%)$ \\
\hline EBRT + systemic & $42(56 \%)$ \\
\hline EBRT + systemic + surgery & $6(8 \%)$ \\
\hline WBRT + systemic \pm surgery & $4(5 \%)$ \\
\hline \multicolumn{2}{|c|}{$\begin{array}{l}\text { GTR = gross-total resection; STR = subtotal resection. } \\
\text { * Missing data: SRS dose }(n=1) \text {; volume }(n=1) \text {; conformity }(n=7) \text {; and } \\
\text { homogeneity }(n=7) \text {. } \\
\text { † The } 15 \text {-Gy treatment included } 2 \text { lesions treated at } 12 \text { and } 13 \text { Gy; the } 18-G y \\
\text { treatment included } 1 \text { lesion treated at } 20 \text { Gy; and the } 24-G y \text { treatment included } \\
1 \text { lesion treated at } 22 \text { Gy. } \\
\text { † Eleven patients }(15 \%) \text { had distant rGBMs with no prior treatment. } \\
\S \text { Excludes biopsies, ancillary surgeries such as shunt placement, and hos- } \\
\text { pice; } 10 \text { patients }(13 \%) \text { had no subsequent procedures prior to salvage SRS. } \\
\text { ๆ Four patients had } 4 \text { subsequent procedures, } 5 \text { had } 5 \text {, and } 1 \text { each had } 6 \text { and } 7 \\
\text { ** Seven patients }(9 \%) \text { had no overall procedures, and } 6 \text { patients }(8 \%) \text { had } \\
\text { other treatments. }\end{array}$} \\
\hline
\end{tabular}

Two factors, total tumor volume and homogeneity index, were seen to be independent prognostic factors for PFS. As with OS, by counting the number of poor prognostic features present (total volume $\geq 15 \mathrm{~cm}^{3}$, homogeneity index [minimum if $>1$ lesion treated] $\leq 1.75$ ), 3 prog- 
TABLE 3. Univariable analysis showing factors significantly associated with OS and PFS

\begin{tabular}{|c|c|c|c|c|}
\hline \multirow[b]{2}{*}{ Factor } & \multicolumn{2}{|l|}{ OS } & \multicolumn{2}{|l|}{ PFS } \\
\hline & Median $(95 \% \mathrm{Cl})$ or $\mathrm{HR}(95 \% \mathrm{Cl})^{*}$ & $\mathrm{p}$ Value & Median $(95 \% \mathrm{Cl})$ or $\mathrm{HR}(95 \% \mathrm{Cl})^{*}$ & $p$ Value \\
\hline \multicolumn{5}{|l|}{ KPS score } \\
\hline$<80$ & $6.3(4.7-11.0)$ & & $4.1(1.5-5.0)$ & \\
\hline$\geq 80$ & $12.2(7.4-16.0)$ & 0.0007 & $4.3(3.5-5.4)$ & 0.51 \\
\hline \multicolumn{5}{|l|}{ RPA class } \\
\hline Class 3 & $12.2(8.1-52.2)$ & & $3.9(1.0-4.6)$ & \\
\hline Class 4 & $12.2(6.3-14.5)$ & & $4.6(2.0-6.1)$ & \\
\hline Classes $5 \& 6$ & $5.5(4.1-11.5)$ & $0.07 \dagger$ & $4.0(2.4-5.0)$ & $0.85 \dagger$ \\
\hline \multicolumn{5}{|c|}{ Maximum tumor diameter } \\
\hline$<3 \mathrm{~cm}$ & $11.5(8.1-13.6)$ & & $4.7(3.7-10.1)$ & \\
\hline$\geq 3 \mathrm{~cm}$ & $8.9(5.2-12.2)$ & 0.28 & $4.3(2.6-4.6)$ & 0.03 \\
\hline \multicolumn{5}{|l|}{ Total tumor volume } \\
\hline$<15 \mathrm{~cm}^{3}$ & $11.7(7.4-13.8)$ & & $4.4(3.7-6.1)$ & \\
\hline$\geq 15 \mathrm{~cm}^{3}$ & $5.6(2.6-11.8)$ & 0.03 & $3.3(1.5-4.6)$ & 0.03 \\
\hline \multicolumn{5}{|l|}{ p53 staining } \\
\hline$<10 \%$ & $5.4(0.8-N A)$ & & $4.0(0.8-11.0)$ & \\
\hline $10-32 \%$ & $14.2(7.4-51.5)$ & & $6.9(1.0-N A)$ & \\
\hline $33-66 \%$ & $14.5(\mathrm{NA})$ & & NA & \\
\hline$>66 \%$ & $11.2(8.1-13.0)$ & $0.008 \ddagger$ & $3.9(15-13.0)$ & $0.16 \ddagger$ \\
\hline Ki-67 staining & & 0.12 & & 0.62 \\
\hline$<10 \%$ & $5.5(4.7-13.6)$ & & $4.6(1.5-5.4)$ & \\
\hline $10-30 \%$ & $12.2(4.1-16.3)$ & & $4.2(2.5-6.1)$ & \\
\hline$>30 \%$ & $11.8(6.1-14.5)$ & $0.04 \ddagger$ & $4.3(1.5-10.1)$ & $0.36 \ddagger$ \\
\hline Homogeneity index & $0.20(0.06-0.67)$ & 0.009 & $0.06(0.02-0.15)$ & $<0.0001$ \\
\hline$>1.75$ & Reference & & Reference & \\
\hline$\leq 1.75$ & $2.95(1.14-7.65)$ & 0.03 & $3.73(1.65-8.42)$ & 0.002 \\
\hline Isodose line & $1.04(1.00-1.08)$ & 0.04 & $1.06(1.04-1.09)$ & $<0.0001$ \\
\hline
\end{tabular}

$\mathrm{HR}=$ hazard ratio; NA = not applicable.

${ }^{*}$ Hazard ratios $>1(<1)$ indicate that the prognosis decreases (increases) as the value of the factor increases.

$\dagger$ Trend test (class 3 vs 4 vs 5 and 6 ).

$\ddagger<10 \%$ versus $\geq 10 \%$.

TABLE 4. Multivariable analysis showing factors significantly associated with OS and PFS

\begin{tabular}{clc}
\hline \multicolumn{1}{c}{ Factor } & $\mathrm{HR}(95 \% \mathrm{Cl})$ & $\mathrm{p}$ Value \\
\hline PFS & & \\
\hline Total tumor volume $\left(<15[\mathrm{ref}]\right.$ vs $\left.\geq 15 \mathrm{~cm}^{3}\right)$ & $2.96(1.24-7.07)$ & 0.01 \\
\hline Homogeneity index $\left(>1.75[\text { ref] vs } \leq 1.75)^{*}\right.$ & $3.73(1.65-8.42)$ & 0.002 \\
\hline OS & & 0.007 \\
\hline Total tumor volume $\left(<15\left[\right.\right.$ ref] vs $\left.\geq 15 \mathrm{~cm}^{3}\right)$ & $3.78(1.44-9.94)$ & 0.0004 \\
\hline KPS score $(\geq 80[$ ref] vs $<80)$ & $5.18(2.09-12.87)$ & 0.03 \\
\hline Homogeneity index $\left(>1.75[\text { ref] vs } \leq 1.75)^{*}\right.$ & $2.95(1.14-7.65)$ & \\
\hline
\end{tabular}

Analyses were stratified by year of SRS.

* If $>1$ lesion was treated in a session the minimum homogeneity index was used.

nostic groups emerged; however, similar to OS prognostic categories, only 1 patient had both total volume $\geq 15 \mathrm{~cm}^{3}$ and a homogeneity index $\leq 1.75$ (PFS was 1.5 months), and therefore patients with 1 or 2 poor features were combined. The two resulting prognostic groups, labeled "favorable" and "unfavorable," comprised 60\% (27/45) and $40 \%(18 / 45)$ of patients, respectively. The estimated 6-month PFS and median PFS of the favorable group were 


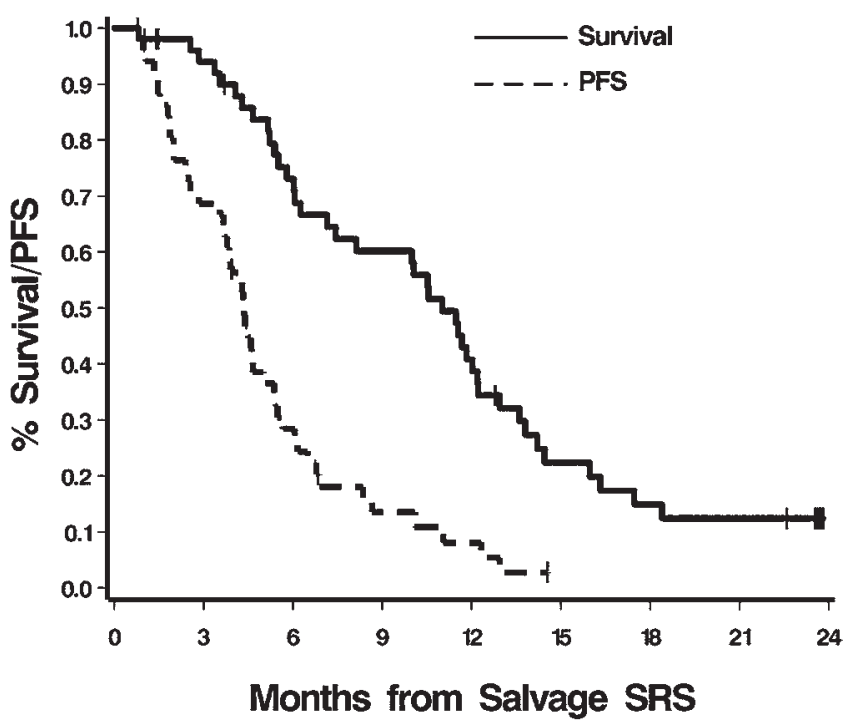

FIG. 1. Kaplan-Meier curves showing the percentage of PFS and OS in patients following SRS for rGBM.

$45 \% \pm 10 \%$ and 5.4 months (95\% CI $4.0-8.4)$, and those for the unfavorable group were $6 \% \pm 5 \%$ and 3.2 months (95\% CI 1.8-4.4), respectively (Table 5 and Fig. 2).

\section{Discussion}

In our study, based on total tumor volume $\left(<15 \mathrm{~cm}^{3}\right)$, homogeneity index ( $>1.75$ ), and KPS score $\geq 80,2$ prognostic groups were identified for PFS, while 3 were identified for OS. The estimated median OS durations for the 3 groups were 15.2 months (95\% CI 7.4-29.0 months), 10.5 months (95\% CI 5.2-12.2 months), and 5.2 months (95\% CI 0.8-6.3 months), respectively. The estimated median PFS of the favorable group was 5.4 months $(95 \%$ CI $4.0-$ 8.4 months), and that for the unfavorable group was 3.2 months (95\% CI 1.8-4.4 months), respectively.

In 2004, Radiation Therapy Oncology Group (RTOG) protocol 93-05 investigated the use of a postoperative SRS boost to any residual tumor followed by EBRT and BCNU versus EBRT and BCNU without the SRS boost in patients with newly diagnosed GBM..$^{51}$ The median OS in the SRS arm was 13.5 months compared to 13.6 months in the non-SRS arm $(p=0.5711) .{ }^{51}$ Since publication of the negative results of this randomized study and those reported by Stupp et al. ${ }^{54}$ in 2005 , the role of SRS in patients with newly diagnosed GBM has not been clearly established. However, the utility of SRS in patients with rGBM was not addressed in these studies. Our previous phase I trial of radiosurgery dose escalation in combination with bevacizumab therapy in patients with rGBM has shown that a single dose of bevacizumab administered about 1 week prior to SRS permitted safe SRS dose escalation of up to 22 Gy for rGBM, with a maximum linear tumor diameter of $2.58 \mathrm{~cm} .{ }^{1}$

\section{Factors Predictive of Outcomes}

Niranjan et al. ${ }^{30}$ reported that younger age ( $<60$ years) and smaller tumor volume $\left(<14 \mathrm{~cm}^{3}\right)$ were associated with improved OS and that smaller tumor volume $(<14$ $\mathrm{cm}^{3}$ ) was associated with improved PFS (4.9 months vs 4.0 months, $p=0.011)$. SRS margin dose was identified as a prognostic factor affecting time to progression in a study in which a low margin dose of 6 Gy was used as a salvage therapy. ${ }^{36}$ Elliott et al. ${ }^{8}$ identified a higher performance score (KPS score $\geq 90$ ), smaller tumor volume, and increased time to recurrence as factors predictive of longer OS. Kong et al. ${ }^{22}$ also identified smaller tumor volume (< $10 \mathrm{~cm}^{3}$ ) as predictive of OS. In the present study, we similarly identified smaller tumor volume $\left(<15 \mathrm{~cm}^{3}\right)$ and KPS score $(\geq 80)$ as independent predictors of improved OS.

The homogeneity index (maximum dose/prescription tumor dose) evaluates the uniformity of the dose distribution in the target volume, with a higher number indicative of a heterogeneous dose distribution that is associated with a greater number of hot spots. Interestingly, we found that a higher homogeneity index ( $>1.75$; i.e., a more heterogeneous plan) was also an independent predictor of both PFS $(p=0.002)$ and OS $(p=0.03)$. Moreover, since the radiation dose is relevant in the management of GBMs, and as Chan et al. ${ }^{4}$ noted, the majority (91\%) of tumor recurrences occurred within the central portion of the tumor volume region (i.e., $95 \%$ of the tumor volume region). These findings of high homogeneity index as an independent, favorable prognostic factor are novel and noteworthy, but they need to be investigated in a large cohort of patients with rGBM managed with salvage SRS.

TABLE 5. Prognostic groups significantly associated with PFS and OS following salvage SRS

\begin{tabular}{ccc}
\hline \multicolumn{1}{c}{ Prognostic Group } & No. of Patients $(\%)$ & Median $(95 \% \mathrm{Cl})$ \\
\hline PFS $(\mathrm{n}=45)$ & & \\
\hline Favorable (homogeneity index $>1.75 \&$ total volume $\left.<15 \mathrm{~cm}^{3}\right)$ & $27(60 \%)$ & $5.4(4.0-8.4)$ \\
\hline Unfavorable (homogeneity index $\leq 1.75 \&$ /or total volume $\left.\geq 15 \mathrm{~cm}^{3}\right)^{\star}$ & $18(40 \%)$ & $3.2(1.8-4.4)$ \\
\hline OS $(\mathrm{n}=44)$ & & $17(39 \%)$ \\
\hline Favorable (no poor prognostic factor) $\dagger$ & $22(50 \%)$ & $15.2(7.4-29.0)$ \\
\hline Intermediate (1 poor prognostic factor) $\dagger$ & $5(11 \%)$ & $5.2(0.8-12.2)$ \\
\hline Unfavorable (2 or 3 prognostic factors) $\dagger$ & & $10.3)$ \\
\hline
\end{tabular}

* Only 1 patient had both a homogeneity index $\leq 1.75$ and volume $\geq 15 \mathrm{~cm}^{3}$; the PFS for this patient was 1.5 months.

$\dagger$ Poor prognostic features: KPS score $<80$, total volume $\geq 15 \mathrm{~cm}^{3}$, and homogeneity index $\leq 1.75$. 

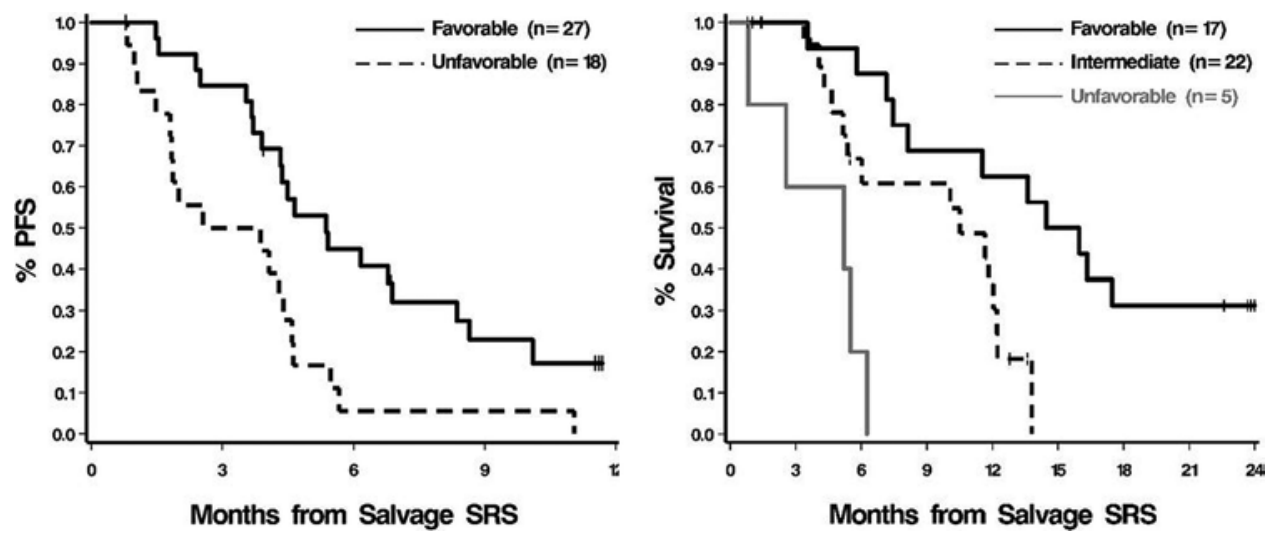

FIG. 2. Kaplan-Meier curves showing PFS (left) in patients stratified into prognostic groups (favorable: homogeneity index $>1.75$ and total volume $<15 \mathrm{~cm}^{3}$; unfavorable: index $\leq 1.75$ and/or total volume $\geq 15 \mathrm{~cm}^{3}$ ) and OS (right) in patients stratified into prognostic groups (favorable: 0 poor prognostic features; intermediate: 1 poor prognostic feature; unfavorable: 2 or 3 poor prognostic features) following salvage SRS for rGBM. The poor prognostic features were KPS score $<80$, total tumor volume $\geq 15 \mathrm{~cm}^{3}$, and homogeneity index $\leq 1.75$.

We also found that patients treated after 2005 had a better outcome than patients treated prior to 2005 (median OS 10.0 vs 11.8 months, $\mathrm{p}=0.05$; median PFS 4.1 vs 4.5 months, $\mathrm{p}=0.05$ ). This may be attributed to the standardization in the management of patients with newly diagnosed GBM following the introduction of Stupp's protocol, which led to improvement in median OS, from 12.1 months to 14.6 months. ${ }^{54}$ However, the difference in our study may also be attributed to higher median prescription dose to a smaller median tumor volume after 2005 (21 Gy to a $0.2-\mathrm{cm}^{3}$ tumor volume) compared to the era prior to 2005 ( 15 Gy to a $10.4-\mathrm{cm}^{3}$ tumor volume, $\mathrm{p}=0.01$ ). Based on these predictive factors, we have created prognostic groups (favorable, intermediate, and unfavorable), which may help guide the selection of patients who are likely to benefit from salvage SRS for rGBM.

In 1997, Kondziolka et al. ${ }^{20}$ reported an overall median survival of 30 months for salvage SRS when performed at the time of recurrence versus 20 months when performed upfront as a part of initial treatment in patients with GBM. Recently, Hsieh et al..$^{14}$ reported an improved median OS of 16.7 months when salvage SRS was performed at the time of tumor progression compared to 10 months when GKRS was performed as an upfront adjunct treatment in 26 patients with GBM using a median margin dose of 12 Gy. This difference in SRS responsiveness in primary versus recurrent GBMs may simply be attributable to selection bias toward patients with good overall performance status for salvage SRS and small local tumor recurrences. Alternatively, perhaps some yet-to-be elucidated factors or features intrinsic to the recurrent tumors contribute to enhanced radiosurgical responsiveness in rGBMs. Further studies regarding optimal timing, SRS dosing, and combination of SRS with other chemotherapeutic/radio-sensitizing agents to achieve maximum efficacy for rGBMs are required.

In our study, patients with a larger number of cells staining for $\mathrm{p} 53$ or Ki-67 showed prolonged OS compared to patients with a lower number of cells staining positive, with no impact on PFS. This finding may be attributed to the fact that focal tumors with rapidly dividing cells are perhaps more likely to respond to SRS than otherwise. Of note, while there was no correlation between the molecular status and overall outcome in our study, due to the small number of patients with available molecular information, the associated statistical power is acknowledged to be low, and we believe further research in this area is needed.

Recently, minimally invasive techniques such as LITT have been shown to be efficacious in managing patients with rGBM. ${ }^{46-48,60}$ The minimally invasive nature of this treatment modality makes it a useful tool, particularly in patients with deep-seated recurrent tumors. In our series, only one patient with one lesion was treated with LITT prior to SRS. The impact of LITT in combination with SRS still needs to be elucidated in cases of rGBM. Similarly, various immunotherapeutic agents ${ }^{15,37,41}$ have been tried in patients with rGBM, and the results have varied. The impact of these agents on SRS outcomes in patients with rGBM could not be evaluated in our study due to heterogeneous treatment modalities and the small sample size. However, this question can be addressed and answered in future studies.

Our finding of a median OS of 11 months after salvage SRS in patients with rGBM appears comparable to the 11.8 months observed following a combination of the newer treatment modality of tumor treating fields and chemotherapy in patients with $\mathrm{rGBM}{ }^{18}$ Moreover, the median OS of 15.2 months in our patients with favorable prognostic factors is very encouraging given that SRS is a wellestablished treatment modality with the convenience of a single treatment session. Our work suggests that SRS is an effective treatment modality in patients with rGBM and may be considered earlier and more often in carefully selected patients (Fig. 3).

\section{Limitations and Future Directions}

The retrospective design, heterogeneous management strategies, potential for selection bias, and lack of control 

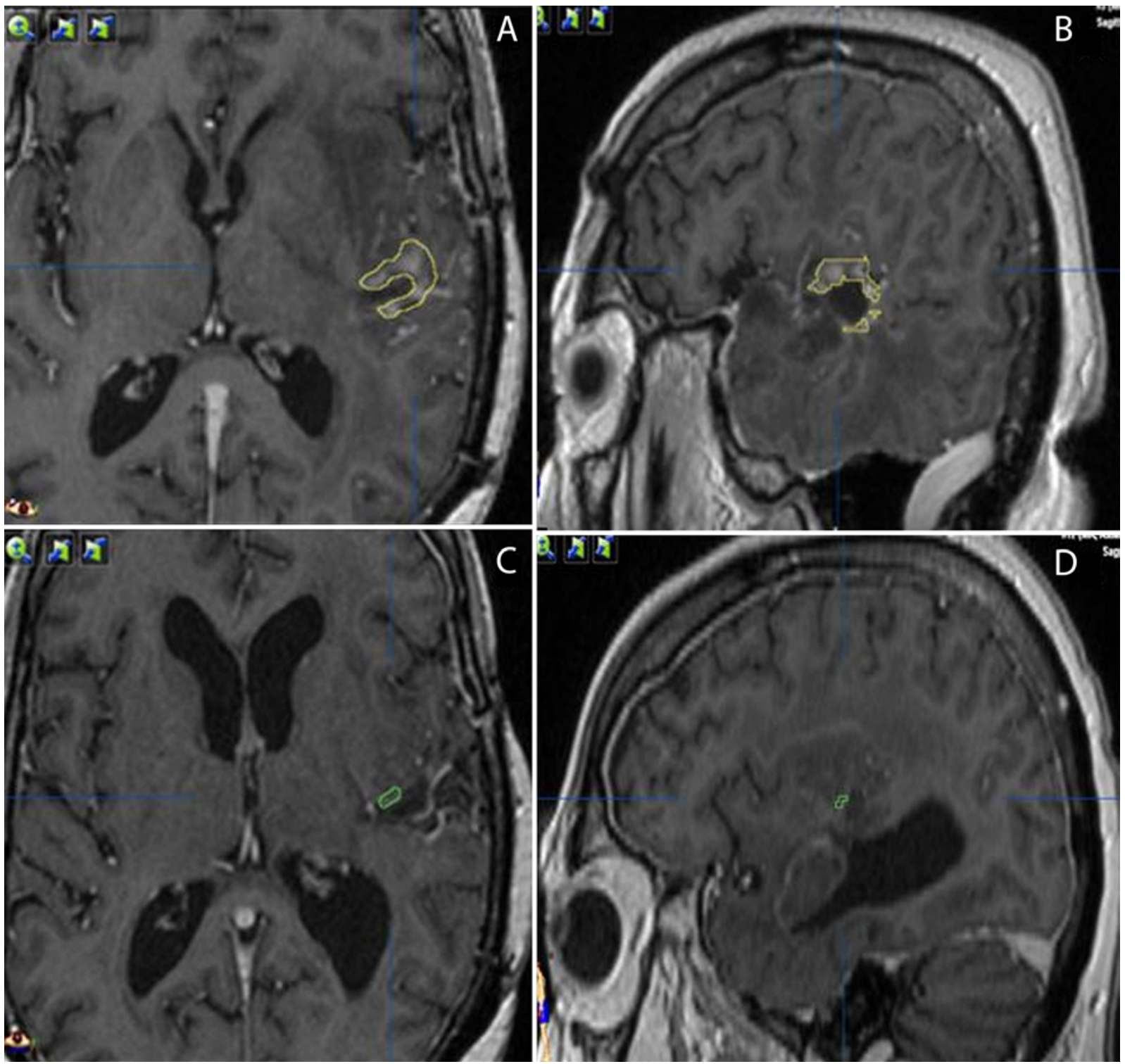

FIG. 3. Illustrative case of a 61-year-old man who presented with rGBM following standard concurrent chemoradiotherapy and 3 cycles of adjunct chemotherapy. He exhibited a good response to salvage SRS (22 Gy at 50\% isodose line; homogeneity index 2.005) at the 9.4-month follow-up (pretreatment axial [A] and sagittal [B] postgadolinium T1-weighted MR images and postgadolinium axial [C] and sagittal [D] T1-weighted MR images at last follow-up). Figure is available in color online only.

groups are potential limitations of our study. Also, the effects of ongoing systemic treatment cannot be segregated from that of local control offered by SRS on follow-up imaging (as in other studies). However, our study confirms the utility of SRS in managing patients with rGBM and identifies prognostic groups. The defined prognostic groups may be a useful tool for clinical decision-making, treatment planning, resource utilization, and clinical trial design.

\section{Conclusions}

Good performance, smaller tumor volumes, and treatment at higher homogeneity indices were associated with longer OS and/or PFS despite multiple prior treatments for
rGBM. SRS for rGBM is a reasonable salvage treatment option for these patients.

\section{References}

1. Abbassy M, Missios S, Barnett GH, Brewer C, Peereboom DM, Ahluwalia M, et al: Phase I trial of radiosurgery dose escalation plus bevacizumab in patients with recurrent/ progressive glioblastoma. Neurosurgery [epub ahead of print], 2017

2. Batchelor TT, Mulholland P, Neyns B, Nabors LB, Campone $\mathrm{M}$, Wick A, et al: Phase III randomized trial comparing the efficacy of cediranib as monotherapy, and in combination with lomustine, versus lomustine alone in patients with recurrent glioblastoma. J Clin Oncol 31:3212-3218, 2013

3. Biswas T, Okunieff P, Schell MC, Smudzin T, Pilcher WH, 
Bakos RS, et al: Stereotactic radiosurgery for glioblastoma: retrospective analysis. Radiat Oncol 4:11, 2009

4. Chan JL, Lee SW, Fraass BA, Normolle DP, Greenberg HS, Junck LR, et al: Survival and failure patterns of high-grade gliomas after three-dimensional conformal radiotherapy. J Clin Oncol 20:1635-1642, 2002

5. Chao ST, Ahluwalia MS, Barnett GH, Stevens GH, Murphy ES, Stockham AL, et al: Challenges with the diagnosis and treatment of cerebral radiation necrosis. Int J Radiat Oncol Biol Phys 87:449-457, 2013

6. Combs SE, Edler L, Rausch R, Welzel T, Wick W, Debus J: Generation and validation of a prognostic score to predict outcome after re-irradiation of recurrent glioma. Acta Oncol 52:147-152, 2013

7. Ellingson BM, Kim HJ, Woodworth DC, Pope WB, Cloughesy $\mathrm{JN}$, Harris RJ, et al: Recurrent glioblastoma treated with bevacizumab: contrast-enhanced T1-weighted subtraction maps improve tumor delineation and aid prediction of survival in a multicenter clinical trial. Radiology 271:200-210, 2014

8. Elliott RE, Parker EC, Rush SC, Kalhorn SP, Moshel YA, Narayana A, et al: Efficacy of Gamma Knife radiosurgery for small-volume recurrent malignant gliomas after initial radical resection. World Neurosurg 76:128-140, 61-62, 2011

9. Field KM, Simes J, Nowak AK, Cher L, Wheeler H, Hovey EJ, et al: Randomized phase 2 study of carboplatin and bevacizumab in recurrent glioblastoma. Neuro Oncol 17:1504-1513, 2015

10. Fisher JL, Schwartzbaum JA, Wrensch M, Wiemels JL: Epidemiology of brain tumors. Neurol Clin 25:867-890, vii, 2007

11. Flickinger JC, Kondziolka D, Lunsford LD, Coffey RJ, Goodman ML, Shaw EG, et al: A multi-institutional experience with stereotactic radiosurgery for solitary brain metastasis. Int J Radiat Oncol Biol Phys 28:797-802, 1994

12. Flieger M, Ganswindt U, Schwarz SB, Kreth FW, Tonn $\mathrm{JC}$, la Fougère $\mathrm{C}$, et al: Re-irradiation and bevacizumab in recurrent high-grade glioma: an effective treatment option. J Neurooncol 117:337-345, 2014

13. Friedman HS, Prados MD, Wen PY, Mikkelsen T, Schiff D, Abrey LE, et al: Bevacizumab alone and in combination with irinotecan in recurrent glioblastoma. J Clin Oncol 27:47334740, 2009

14. Hsieh PC, Chandler JP, Bhangoo S, Panagiotopoulos K, Kalapurakal JA, Marymont MH, et al: Adjuvant gamma knife stereotactic radiosurgery at the time of tumor progression potentially improves survival for patients with glioblastoma multiforme. Neurosurgery 57:684-692, 2005

15. Hunn MK, Bauer E, Wood CE, Gasser O, Dzhelali M, Ancelet LR, et al: Dendritic cell vaccination combined with temozolomide retreatment: results of a phase I trial in patients with recurrent glioblastoma multiforme. J Neurooncol 121:319-329, 2015

16. Hunter GK, Suh JH, Reuther AM, Vogelbaum MA, Barnett $\mathrm{GH}$, Angelov L, et al: Treatment of five or more brain metastases with stereotactic radiosurgery. Int J Radiat Oncol Biol Phys 83:1394-1398, 2012

17. Kano H, Urgosik D, Liscak R, Pollock BE, Cohen-Inbar O, Sheehan JP, et al: Stereotactic radiosurgery for idiopathic glossopharyngeal neuralgia: an international multicenter study. J Neurosurg 125 (Suppl 1):147-153, 2016

18. Kesari S, Ram Z: Tumor-treating fields plus chemotherapy versus chemotherapy alone for glioblastoma at first recurrence: a post hoc analysis of the EF-14 trial. CNS Oncol 6:185-193, 2017

19. Kim M, Lee DH, Kim HJ, Cho YH, Kim JH, Kwon DH: Analysis of the results of recurrent intracranial meningiomas treated with re-radiosurgery. Clin Neurol Neurosurg 153:93-101, 2017

20. Kondziolka D, Flickinger JC, Bissonette DJ, Bozik M,
Lunsford LD: Survival benefit of stereotactic radiosurgery for patients with malignant glial neoplasms. Neurosurgery 41:776-785, 1997

21. Kondziolka D, Patel A, Lunsford LD, Kassam A, Flickinger JC: Stereotactic radiosurgery plus whole brain radiotherapy versus radiotherapy alone for patients with multiple brain metastases. Int J Radiat Oncol Biol Phys 45:427-434, 1999

22. Kong DS, Lee JI, Park K, Kim JH, Lim DH, Nam DH: Efficacy of stereotactic radiosurgery as a salvage treatment for recurrent malignant gliomas. Cancer 112:2046-2051, 2008

23. Kreisl TN, Kim L, Moore K, Duic P, Royce C, Stroud I, et al: Phase II trial of single-agent bevacizumab followed by bevacizumab plus irinotecan at tumor progression in recurrent glioblastoma. J Clin Oncol 27:740-745, 2009

24. Kruyt IJ, Verheul JB, Hanssens PEJ, Kunst HPM: Gamma Knife radiosurgery for treatment of growing vestibular schwannomas in patients with neurofibromatosis Type 2: a matched cohort study with sporadic vestibular schwannomas. J Neurosurg 128:49-59, 2018

25. Lee EW, Wei LJ, Amato DA, Leurgans S: Cox-type regression analysis for large numbers of small groups of correlated failure time observations, in Klein JP, Goel PK (eds): Survival Analysis: State of the Art. Dordrecht: Springer Netherlands, 1992, pp 237-247

26. Li J, Wang M, Won M, Shaw EG, Coughlin C, Curran WJ Jr, et al: Validation and simplification of the Radiation Therapy Oncology Group recursive partitioning analysis classification for glioblastoma. Int J Radiat Oncol Biol Phys 81:623-630, 2011

27. Lin NU, Lee EQ, Aoyama H, Barani IJ, Barboriak DP, Baumert BG, et al: Response assessment criteria for brain metastases: proposal from the RANO group. Lancet Oncol 16:e270-e278, 2015

28. Minniti G, Agolli L, Falco T, Scaringi C, Lanzetta G, Caporello P, et al: Hypofractionated stereotactic radiotherapy in combination with bevacizumab or fotemustine for patients with progressive malignant gliomas. J Neurooncol 122:559566, 2015

29. Nagpal J, Jamoona A, Gulati ND, Mohan A, Braun A, Murali $\mathrm{R}$, et al: Revisiting the role of p53 in primary and secondary glioblastomas. Anticancer Res 26 (6C):4633-4639, 2006

30. Niranjan A, Kano H, Iyer A, Kondziolka D, Flickinger JC, Lunsford LD: Role of adjuvant or salvage radiosurgery in the management of unresected residual or progressive glioblastoma multiforme in the pre-bevacizumab era. J Neurosurg 122:757-765, 2015

31. Ostrom QT, Gittleman H, Xu J, Kromer C, Wolinsky Y, Kruchko C, et al: CBTRUS Statistical Report: Primary brain and other central nervous system tumors diagnosed in the United States in 2009-2013. Neuro Oncol 18 (Suppl 5):v1v75, 2016

32. Park KJ, Kano H, Iyer A, Liu X, Niranjan A, Flickinger JC, et al: Salvage gamma knife stereotactic radiosurgery followed by bevacizumab for recurrent glioblastoma multiforme: a case-control study. J Neurooncol 107:323-333, 2012

33. Patel M, Siddiqui F, Jin JY, Mikkelsen T, Rosenblum M, Movsas B, et al: Salvage reirradiation for recurrent glioblastoma with radiosurgery: radiographic response and improved survival. J Neurooncol 92:185-191, 2009

34. Patil CG, Pricola K, Sarmiento JM, Garg SK, Bryant A, Black KL: Whole brain radiation therapy (WBRT) alone versus WBRT and radiosurgery for the treatment of brain metastases. Cochrane Database Syst Rev (9):CD006121, 2012

35. Perry JR, Bélanger K, Mason WP, Fulton D, Kavan P, Easaw J, et al: Phase II trial of continuous dose-intense temozolomide in recurrent malignant glioma: RESCUE study. J Clin Oncol 28:2051-2057, 2010

36. Pouratian N, Crowley RW, Sherman JH, Jagannathan J, 
Sheehan JP: Gamma Knife radiosurgery after radiation therapy as an adjunctive treatment for glioblastoma. J Neurooncol 94:409-418, 2009

37. Reardon DA, Desjardins A, Schuster J, Tran DD, Fink KL, Nabors LB, et al: IMCT-08 ReACT: long-term survival from a randomized phase II study of rindopepimut (CDX-110) plus bevacizumab in relapsed glioblastoma. Neuro Oncol 17 (Suppl 5):v109, 2015 (Abstract)

38. Reardon DA, Nabors LB, Mason WP, Perry JR, Shapiro W, Kavan P, et al: Phase I/randomized phase II study of afatinib, an irreversible ErbB family blocker, with or without protracted temozolomide in adults with recurrent glioblastoma. Neuro Oncol 17:430-439, 2015

39. Ryken TC, McDermott M, Robinson PD, Ammirati M, Andrews DW, Asher AL, et al: The role of steroids in the management of brain metastases: a systematic review and evidence-based clinical practice guideline. J Neurooncol 96:103-114, 2010

40. Ryu S, Buatti JM, Morris A, Kalkanis SN, Ryken TC, Olson $\mathrm{JJ}$ : The role of radiotherapy in the management of progressive glioblastoma: a systematic review and evidence-based clinical practice guideline. J Neurooncol 118:489-499, 2014

41. Sampson J, Omuro A, Vlahovic G, Sahebjam S, Baehring J, Hafler DA, et al: IMCT-03 Safety and activity of nivolumab monotherapy and nivolumab in combination with ipilimumab in recurrent glioblastoma: updated results from CHECKMATE-143. Neuro Oncol 17 (Suppl 5):v107, 2015 (Abstract)

42. Seystahl K, Wick W, Weller M: Therapeutic options in recurrent glioblastoma-an update. Crit Rev Oncol Hematol 99:389-408, 2016

43. Sharma M, Jia X, Ahluwalia M, Barnett GH, Vogelbaum MA, Chao ST, et al: Cumulative intracranial tumor volume and number of brain metastasis as predictors of developing new lesions after stereotactic radiosurgery for brain metastasis. World Neurosurg 106:666-675, 2017

44. Sharma M, Jia X, Ahluwalia M, Barnett GH, Vogelbaum MA, Chao ST, et al: First follow-up radiographic response is one of the predictors of local tumor progression and radiation necrosis after stereotactic radiosurgery for brain metastases. Cancer Med 6:2076-2086, 2017

45. Shaw E, Scott C, Souhami L, Dinapoli R, Kline R, Loeffler $\mathrm{J}$, et al: Single dose radiosurgical treatment of recurrent previously irradiated primary brain tumors and brain metastases: final report of RTOG protocol 90-05. Int J Radiat Oncol Biol Phys 47:291-298, 2000

46. Silva D, Sharma M, Barnett GH: Laser ablation vs open resection for deep-seated tumors: evidence for laser ablation. Neurosurgery 63 (Suppl 1):15-26, 2016

47. Silva D, Sharma M, Belsuzarri T, Barnett GH: Minimally invasive targeted therapy for glioblastoma: laser interstitial thermal therapy, in Brem S, Abdullah KG (eds): Glioblastoma. Philadelphia: Elsevier, 2017

48. Silva D, Sharma M, Juthani R, Meola A, Barnett GH: Magnetic resonance thermometry and laser interstitial thermal therapy for brain tumors. Neurosurg Clin N Am 28:525-533, 2017

49. Skeie BS, Enger PO, Brøgger J, Ganz JC, Thorsen F, Heggdal JI, et al: Gamma Knife surgery versus reoperation for recurrent glioblastoma multiforme. World Neurosurg 78:658-669, 2012

50. Soon YY, Tham IW, Lim KH, Koh WY, Lu JJ: Surgery or radiosurgery plus whole brain radiotherapy versus surgery or radiosurgery alone for brain metastases. Cochrane Database Syst Rev (3):CD009454, 2014

51. Souhami L, Seiferheld W, Brachman D, Podgorsak EB, Werner-Wasik M, Lustig R, et al: Randomized comparison of stereotactic radiosurgery followed by conventional radiotherapy with carmustine to conventional radiotherapy with carmustine for patients with glioblastoma multiforme: report of Radiation Therapy Oncology Group 93-05 protocol. Int J Radiat Oncol Biol Phys 60:853-860, 2004

52. Stupp R, Hegi ME, Mason WP, van den Bent MJ, Taphoorn MJ, Janzer RC, et al: Effects of radiotherapy with concomitant and adjuvant temozolomide versus radiotherapy alone on survival in glioblastoma in a randomised phase III study: 5-year analysis of the EORTC-NCIC trial. Lancet Oncol 10:459-466, 2009

53. Stupp R, Idbaih A, Steinberg DM, Read W, Toms S, Barnett G, et al: LTBK-01: Prospective, multi-center phase III trial of tumor treating fields together with temozolomide compared to temozolomide alone in patients with newly diagnosed glioblastoma. Neuro Oncol 18 (Suppl 6):i1, 2016 (Abstract)

54. Stupp R, Mason WP, van den Bent MJ, Weller M, Fisher B, Taphoorn MJ, et al: Radiotherapy plus concomitant and adjuvant temozolomide for glioblastoma. N Engl J Med 352:987-996, 2005

55. Stupp R, Taillibert S, Kanner AA, Kesari S, Steinberg DM, Toms SA, et al: Maintenance therapy with tumortreating fields plus temozolomide vs temozolomide alone for glioblastoma: a randomized clinical trial. JAMA 314:25352543, 2015

56. Stupp R, Wong ET, Kanner AA, Steinberg D, Engelhard H, Heidecke V, et al: NovoTTF-100A versus physician's choice chemotherapy in recurrent glioblastoma: a randomised phase III trial of a novel treatment modality. Eur J Cancer 48:2192-2202, 2012

57. Suchorska B, Weller M, Tabatabai G, Senft C, Hau P, Sabel $\mathrm{MC}$, et al: Complete resection of contrast-enhancing tumor volume is associated with improved survival in recurrent glioblastoma-results from the DIRECTOR trial. Neuro Oncol 18:549-556, 2016

58. Sughrue ME, Sheean T, Bonney PA, Maurer AJ, Teo C: Aggressive repeat surgery for focally recurrent primary glioblastoma: outcomes and theoretical framework. Neurosurg Focus 38(3):E11, 2015

59. Taal W, Oosterkamp HM, Walenkamp AM, Dubbink HJ, Beerepoot LV, Hanse MC, et al: Single-agent bevacizumab or lomustine versus a combination of bevacizumab plus lomustine in patients with recurrent glioblastoma (BELOB trial): a randomised controlled phase 2 trial. Lancet Oncol 15:943-953, 2014

60. Thomas JG, Rao G, Kew Y, Prabhu SS: Laser interstitial thermal therapy for newly diagnosed and recurrent glioblastoma. Neurosurg Focus 41(4):E12, 2016

61. Torok JA, Wegner RE, Mintz AH, Heron DE, Burton SA: Re-irradiation with radiosurgery for recurrent glioblastoma multiforme. Technol Cancer Res Treat 10:253-258, 2011

62. Trifiletti DM, Lee CC, Kano H, Cohen J, Janopaul-Naylor J, Alonso-Basanta M, et al: Stereotactic radiosurgery for brainstem metastases: an international cooperative study to define response and toxicity. Int J Radiat Oncol Biol Phys 96:280-288, 2016

63. Tsao MN, Mehta MP, Whelan TJ, Morris DE, Hayman JA, Flickinger JC, et al: The American Society for Therapeutic Radiology and Oncology (ASTRO) evidence-based review of the role of radiosurgery for malignant glioma. Int J Radiat Oncol Biol Phys 63:47-55, 2005

64. Valery CA, Faillot M, Lamproglou I, Golmard JL, Jenny C, Peyre M, et al: Grade II meningiomas and Gamma Knife radiosurgery: analysis of success and failure to improve treatment paradigm. J Neurosurg 125 (Suppl 1):89-96, 2016

65. van den Bent MJ, Brandes AA, Rampling R, Kouwenhoven MC, Kros JM, Carpentier AF, et al: Randomized phase II trial of erlotinib versus temozolomide or carmustine in recurrent glioblastoma: EORTC brain tumor group study 26034. J Clin Oncol 27:1268-1274, 2009

66. Weller M, Tabatabai G, Kästner B, Felsberg J, Steinbach 
JP, Wick A, et al: MGMT promoter methylation is a strong prognostic biomarker for benefit from dose-intensified temozolomide rechallenge in progressive glioblastoma: the DIRECTOR trial. Clin Cancer Res 21:2057-2064, 2015

67. Wick W, Brandes AA, Gorlia T, Bendszus M, Sahm F, Taal W, et al: LB-05 Phase III trial exploring the combination of bevacizumab and lomustine in patients with first recurrence of a glioblastoma: the EORTC 26101 trial. Neuro Oncol 17 (Suppl 5):v1, 2015 (Abstract)

68. Yamamoto M, Serizawa T, Shuto T, Akabane A, Higuchi Y, Kawagishi J, et al: Stereotactic radiosurgery for patients with multiple brain metastases (JLGK0901): a multi-institutional prospective observational study. Lancet Oncol 15:387-395, 2014

69. Zeng A, Hu Q, Liu Y, Wang Z, Cui X, Li R, et al: IDH1/2 mutation status combined with Ki-67 labeling index defines distinct prognostic groups in glioma. Oncotarget 6:3023230238, 2015

\section{Disclosures}

Dr. Barnett is a consultant for Elekta. Dr. Mohammadi is a consultant for Monteris Medical.

\section{Author Contributions}

Conception and design: Angelov, Sharma. Acquisition of data: Sharma, Schroeder, Meola. Analysis and interpretation of data: Sharma, Elson. Drafting the article: Sharma. Critically revising the article: Angelov, Sharma, Barnett, Vogelbaum, Suh, Chao, Mohammadi, Stevens, Murphy. Reviewed submitted version of manuscript: Angelov, Sharma, Schroeder, Meola, Barnett, Vogelbaum, Suh, Chao, Mohammadi, Stevens, Murphy. Approved the final version of the manuscript on behalf of all authors: Angelov. Statistical analysis: Elson. Administrative/technical/material support: Angelov, Barnett. Study supervision: Angelov, Barnett.

\section{Correspondence}

Lilyana Angelov: Cleveland Clinic, Cleveland, OH. angelol@ ccf.org. 